\title{
SH003 represses tumor angiogenesis by blocking VEGF binding to VEGFR2
}

\author{
Hyeong Sim Choi ${ }^{1}$, Min Kyoung Kim${ }^{1}$, Kangwook Lee ${ }^{1}$, Kang Min Lee ${ }^{1}$, Youn Kyung \\ $\mathrm{Choi}^{2}$, Yong Cheol Shin ${ }^{3}$, Sung-Gook $\mathrm{Cho}^{4}$, Seong-Gyu Ko ${ }^{3}$ \\ ${ }^{1}$ Department of Science in Korean Medicine, Graduate School, Kyung Hee University, Seoul, Korea \\ ${ }^{2}$ Jeju International Marine Science Center for Research and Education, Korea Institute of Ocean Science \& Technology \\ (KIOST), Jeju, Korea \\ ${ }^{3}$ Department of Preventive Medicine, College of Korean Medicine, Kyung Hee University, Seoul, Korea \\ ${ }^{4}$ Department of Biotechnology, Korea National University of Transportation, Jeungpyeong, Chungbuk, Korea \\ Correspondence to: Sung-Gook Cho, e-mail: chosg@ut.ac.kr \\ Seong-Gyu Ko, e-mail: epiko@khu.ac.kr
}

Keywords: SH003, tumor angiogenesis, VEGF, VEGFR2, TCM

Received: December 18, $2015 \quad$ Accepted: March 31, $2016 \quad$ Published: April 18, 2016

\section{ABSTRACT}

Tumor angiogenesis is a key feature of cancer progression, because a tumor requires abundant oxygen and nutrition to grow. Here, we demonstrate that $\mathrm{SHOO3}_{\text {, }}$ a mixed herbal extract containing Astragalus membranaceus (Am), Angelica gigas (Ag) and Trichosanthes Kirilowii Maximowicz (Tk), represses VEGF-induced tumor angiogenesis both in vitro and in vivo. SHOO3 inhibited VEGF-induced migration, invasion and tube formation in human umbilical vein endothelial cells (HUVEC) with no effect on the proliferation. SH003 reduced CD31-positive vessel numbers in tumor tissues and retarded tumor growth in our xenograft mouse tumor model, while SH003 did not affect pancreatic tumor cell viability. Consistently, SH003 inhibited VEGFstimulated vascular permeability in ears and back skins. Moreover, SH003 inhibited VEGF-induced VEGFR2-dependent signaling by blocking VEGF binding to VEGFR2. Therefore, our data conclude that $\mathrm{SHOO3}$ represses tumor angiogenesis by inhibiting VEGF-induced VEGFR2 activation, and suggest that SH003 may be useful for treating cancer.

\section{INTRODUCTION}

Traditional Chinese herbal medicines have long been used to treat diseases including cancer, while effective chemical components have not been clearly elucidated [1-12]. SH003 is a mixed herbal extract containing Astragalus membranaceus (Am), Angelica gigas (Ag), and Trichosanthes Kirilowii Maximowicz (Tk), which is based on the principle of traditional Chinese medicine [12-16]. Each herbal component has been revealed to have anti-cancer effects, and our resent study has shown that a mixture of those components named $\mathrm{SH} 003$ was better than each component in anti-breast cancer effect [17-22].

Tumor angiogenesis is an essential process for cancer progression, as angiogenic vessels supply nutrients and oxygen to the tumor $[23,24]$. Vascular endothelial cells (ECs) in the angiogenic process move toward the tumor and form new vessels [25, 26]. Vascular endothelial growth factor (VEGF) is mainly released from tumor cells and targets VEGF receptor 2 (VEGFR2), which is a crucial paracrine path for tumor angiogenesis [27]. VEGFinduced VEGFR2-mediated signaling highly expresses matrix metallopeptidase-9 (MMP-9), promoting a directional migration of ECs [28-30]. Therefore, targeting VEGF-induced VEGFR2-mediated signaling in ECs is important for cancer treatment [24, 31-33].

Our previous study found that $\mathrm{SH} 003$ reduced CD31-stained vessel numbers in tumor tissues [13], which raised a question of whether $\mathrm{SH} 003$ directly affects tumor angiogenesis. Ag inhibits VEGF-induced angiogenesis, while Am induces angiogenesis [18, 34-36]. In addition, an anti-angiogenic effect of Tk is not reported. Accordingly, we hypothesized that SH003 might have a therapeutic usage in inhibiting tumor angiogenesis.

Our in vitro and in vivo studies demonstrate that SH003 inhibits VEGF-induced tumor angiogenesis by 
blocking VEGFR2-mediated signaling, which suggest that metronomic SH003 treatment could be useful for treating cancer.

\section{RESULTS}

\section{SH003 inhibits VEGF-stimulated human endothelial cell migration, invasion and tube formation}

We first investigated whether SH003 inhibits in vitro VEGF-dependent angiogenic processes in human endothelial cells such as proliferation, migration, invasion and tube formation, as those cellular aspects are basically investigated in the in vitro angiogenesis [33, 37-39]. To measure an effect of $\mathrm{SH} 003$ on the proliferation, human umbilical vascular endothelial cells (HUVECs) were treated with VEGF $(50 \mathrm{ng} / \mathrm{ml})$ and different concentrations of SH003 $(10,20$ or $50 \mu \mathrm{g} / \mathrm{ml})$ for 24 hours. While VEGF increased HUVEC proliferation rate as previously reported [38, 40], SH003 did not affect VEGF-induced HUVEC proliferation (Figure 1A). However, SH003 inhibited the number of VEGF-dependent migrated cells into the scratched region, when cells were treated with VEGF and SH003 for 9.5 hours (Figure 1B). Likewise, SH003 inhibited the HUVEC invasive ability induced by VEGF, as SH003 reduced invaded cell numbers in dose-dependent manner (Figure 1C). Moreover, SH003 inhibited VEGF-dependent tube formation on matrigels (Figure 1D). Therefore, our data suggest that SH003 effectively inhibits VEGF-dependent cell migration, invasion, and tube formation of the endothelial cells but not the proliferation.

\section{SH003 inhibits VEGF-induced tumor angiogenesis in vivo}

Angiogenesis has a pivotal role in tumor growth and metastasis, and VEGF/VEGFR2-mediated signaling is crucial for tumor angiogenesis [28]. As our data showed that SH003 inhibited VEGF/VEGFR2-mediated angiogenesis in vitro, we further evaluated whether $\mathrm{SH} 003$ represses tumor growth by inhibiting tumor angiogenesis in vivo. SH003 did not inhibit the viability of Panc-28luc pancreatic tumor cells in vitro (Figure 2A). However, SH003 retarded tumor growth in vivo, when Panc-28-luc cells were s.c. injected into the immunodeficient mice, and then added p.o. with SH003 (Figure 2B). Consistently, the average tumor volume was smaller in mice treated with SH003 than in the control (Figure 2C). In addition, SH003 did not affect body weights (Figure 2D), indicating that SH003 at the concentration we used might be safe. The immunohistochemistry confirmed that SH003 inhibited pancreatic tumor growth via tumor angiogenesis. SH003 reduced expression levels of Ki67, p-VEGFR2 and MMP9 , and increased expression level of cleaved caspase-3 in tumor tissues (Figure 2E). Moreover, when we stained tumor tissues with anti-CD31 antibody, and SH003 reduced the vessel numbers (Figure 2E and 2F). Therefore, our data indicate that $\mathrm{SH} 003$ suppresses pancreatic tumor growth by inhibiting tumor angiogenesis.

In addition, we investigated whether SH003 affects VEGF-induced vascular permeability in vivo. SH003 reduced VEGF-induced leakage of Evans blue dye in the ears or back skins of mice (Figure 3A and 3B), indicating that SH003 inhibits VEGF-induced vessel permeability in vivo.

\section{SH003 suppresses VEGF/VEGFR2-mediated angiogenic signaling by blocking VEGF binding to VEGFR2}

Next, we investigated SH003 effect on VEGFdependent VEGFR2-mediated angiogenic signaling. SH003 inhibited VEGF-stimulated intracellular angiogenic signaling in dose-dependent manner, when HUVECs were pretreated with $\mathrm{SH} 003$ at different concentrations for 1 hour and then treated with VEGF for another 1 hour (Figure 4A). SH003 decreased VEGF-dependent VEGFR2 phosphorylation at T1175 and Y1214 residues, resulting in reduction of phosphorylation of FAK, SRC, ERK, AKT, and STAT3. Moreover, when we examined the time-course of SH003 inhibition of VEGF-induced VEGFR2-mediated signaling, SH003 strongly suppressed VEGF-dependent phosphorylation of VEGFR2, FAK, SRC, ERK, AKT, and STAT3 until 2 hours after VEGF stimulation (Figure 4B). Therefore, our data suggest that SH003 has anti-angiogenic effect via inhibiting VEGFstimulated VEGFR2-mediated angiogenic signaling in the endothelial cells.

We next examined whether SH003 affects MMP-9 expression, as VEGF-induced tumor angiogenic vessel cells require MMP-9 to move toward a tumor mass [41, 42]. SH003 inhibited VEGF-induced MMP-9 protein expression in dose- and time-dependent manners (Figure 4C). Accordingly, SH003 inhibited VEGF-induced MMP9 enzymatic activation (Figure 4D).

As SH003 inhibited VEGF-dependent VEGFR2 phosphorylation and its downstream signaling, we further analyzed whether SH003 inhibits an interaction of VEGF with VEGFR2. In our in vitro VEGF-VEGFR2 interaction assays, $\mathrm{SH} 003$ directly inhibited the interaction between VEGF and VEGFR2 (Figure 5A). Moreover, SH003 inhibited the interaction between VEGF and VEGFR1, while the inhibitions showed different $\mathrm{IC}_{50}$ values (Figure 5B). We recently confirmed that $\mathrm{SH} 003$ and its herbal components contained cucurbitacin $\mathrm{D}$, decursin, formononetin, and nodakenin [13]. Therefore, we further examined whether those chemical compounds inhibits VEGF binding to VEGFR2. While different $\mathrm{IC}_{50}$ values were determined, each chemical compound inhibited VEGF binding to VEGFR2 (Figure 5C-5F). Those data 


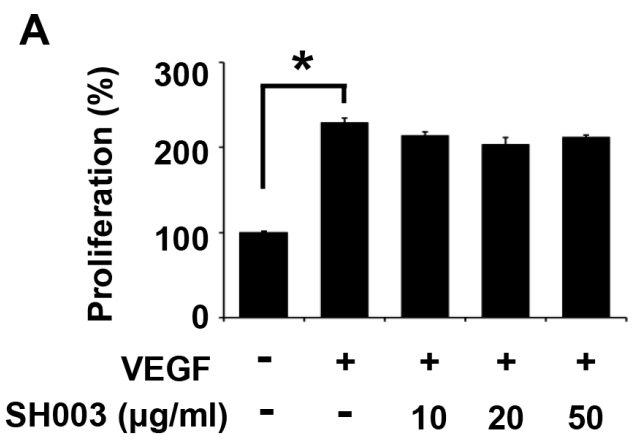

B

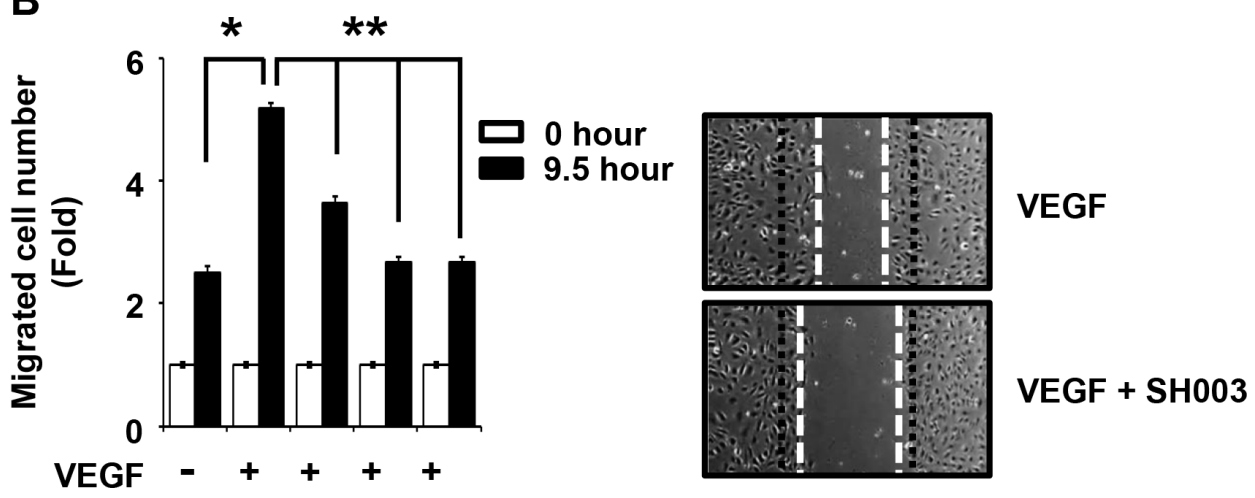

$\mathrm{SH} 003(\mu \mathrm{g} / \mathrm{ml})=-102050$

C
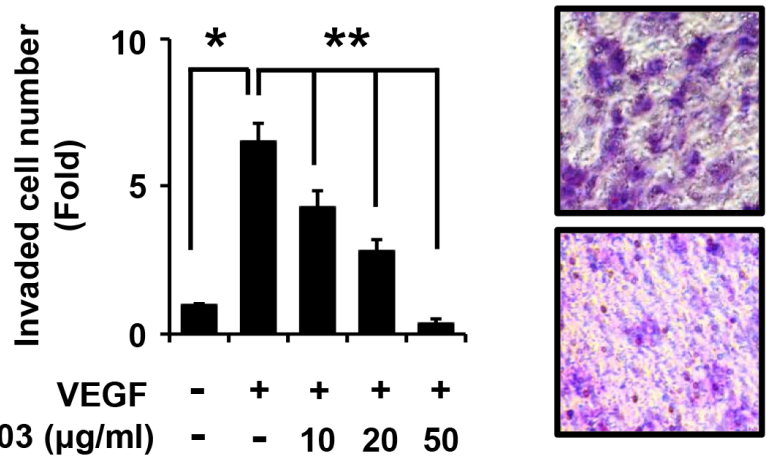

VEGF
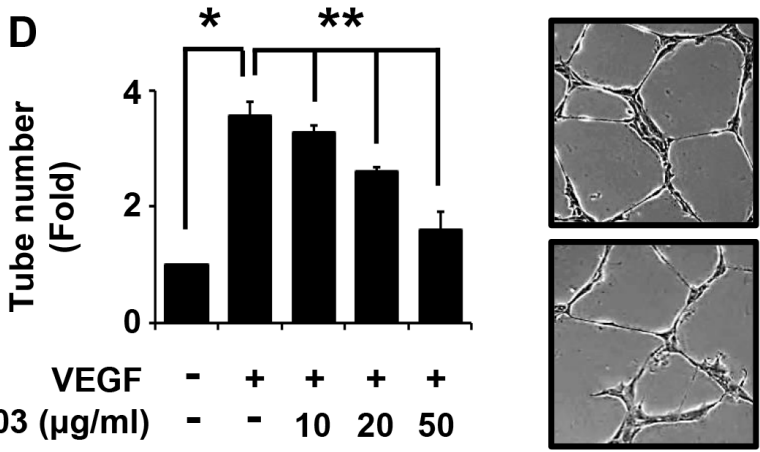

VEGF

VEGF + SH003

Figure 1: SH003 inhibits VEGF-induced angiogenic properties of HUVEC. A. Cell viability in the presence of VEGF and different concentration of SH003 was measured by MTT assay (mean \pm SD; $n=6$ ). B. Cell migration. Left panel, SH003 inhibits VEGFinduced cell migration in wound healing assay. Right panel, representative images of left panel results (x40). C. Cell invasion. Left panel, SH003 inhibits VEGF-induced cell invasion in Boyden chamber assay. Right panel, representative images of left panel results (x40). D. Tube formation. Left panel, SH003 inhibits VEGF-induced tube formation in Matrigel. Right panel, representative images of left panel results $(\mathrm{x} 40){ }^{*}$ and $* *, p<0.05$. 
A

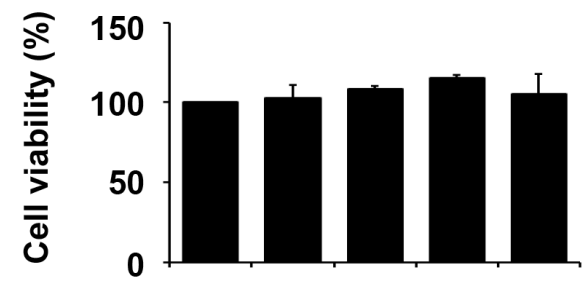

$\mathrm{SH} 003(\mu \mathrm{g} / \mathrm{ml}) \quad 0 \quad 50 \quad 100 \quad 200 \quad 500$

B
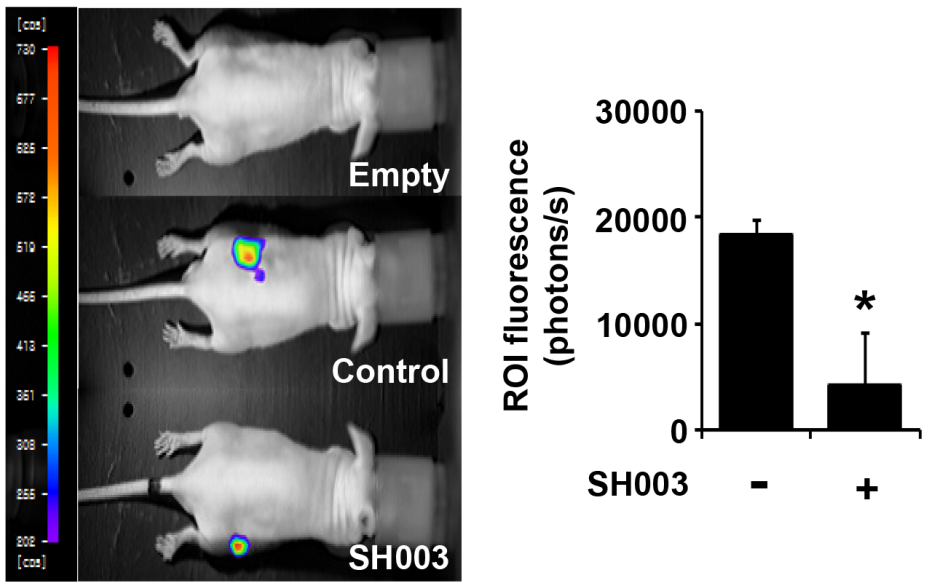

E

C

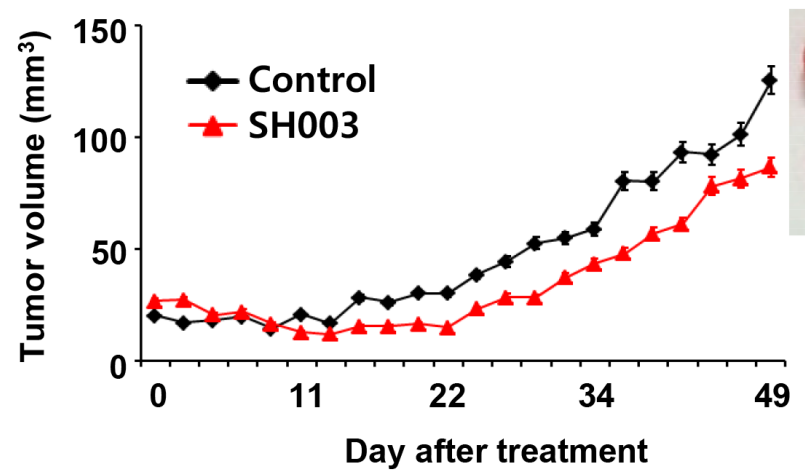

D

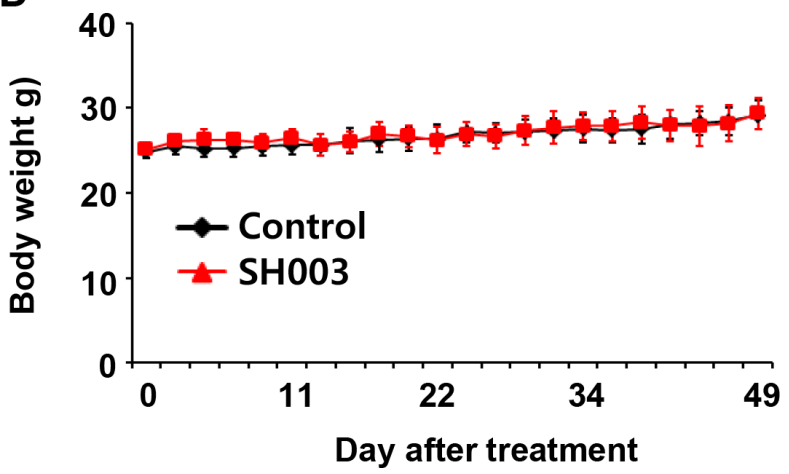

$\mathbf{F}$

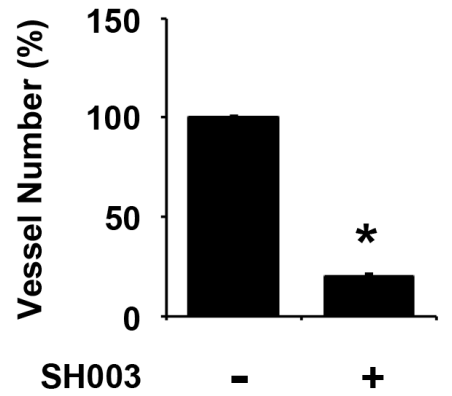

Figure 2: SH003 inhibits tumor growth in vivo. A. The effect of SH003 on the viability of Panc-28-luc cells was determined by the MTT assay (mean $\pm \mathrm{SD} ; n=6$ ). B. Left panel, effects of SH003 on xenograft tumor growth were analyzed by bioluminescence imaging system. Right panel, bars represent quantitative data for left panel results. C-D. SH003 inhibits tumor growth without detectable toxicity. E. Immunohistochemistry of tumor sections. F. The number of CD31-positive vessels in tumor tissues were counted $(n$; control group $=7$ or $\mathrm{SH} 003$ group $=4) .{ }^{*}, p<0.05$. 
suggest that SH003 and its compounds directly inhibit VEGF/VEGFR2 interaction.

\section{DISCUSSION}

VEGF activation of VEGFR2-mediated signaling is a key step for tumor angiogenesis [25, 27, 38, 43-45]. Therefore, the inhibition of VEGF/VEGFR2-mediated angiogenic signaling is one of excellent therapeutic ways to treat cancer growth and/or metastasis [45-47]. In this study, we found that $\mathrm{SH} 003$ inhibited tumor angiogenesis by blocking VEGF binding to VEGFR2.

Our previous study showed that $\mathrm{SH} 003$ at higher concentrations caused breast cancer cell death by inhibiting STAT3-mediated signaling pathway [13]. SH003 concentrations tested in this study was lowered

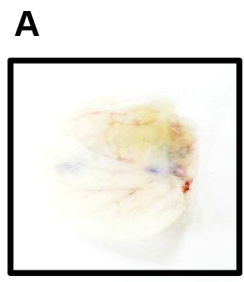

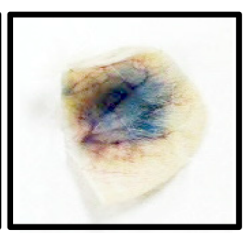

Control

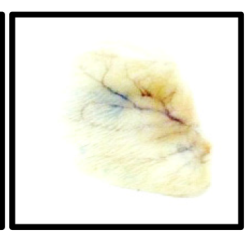

SH003

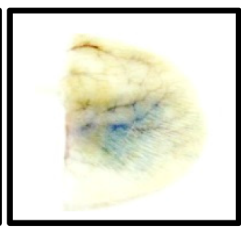

SH003 + VEGF

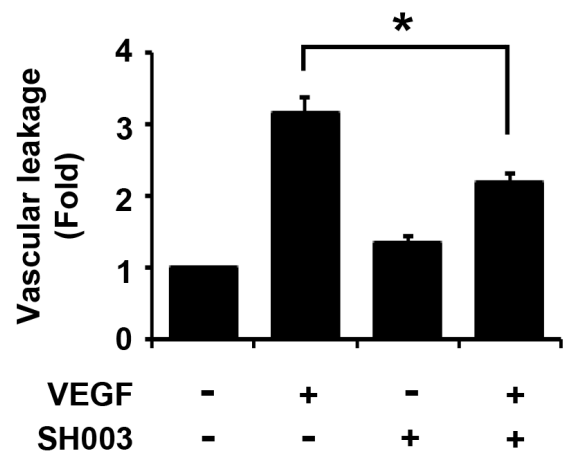

B
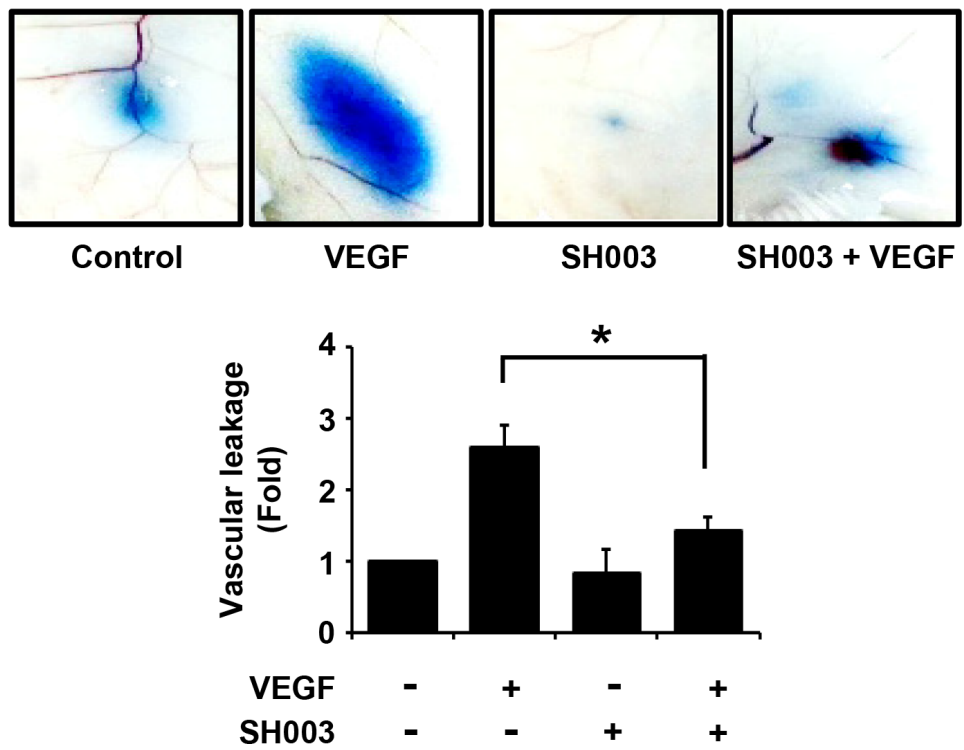

Figure 3: SH003 inhibits VEGF-induced vascular permeability in vivo. A. Top panel, SH003 inhibits VEGF-induced leakage of Evans blue dye into ears of mouse (mean $\pm \mathrm{SD} ; n=5$ ). Bottom panel, data represent quantitative results for top panel results. B. Top panel, in back skins the effect of SH003 on the vascular permeability was determined by the leakage assay (mean $\pm \mathrm{SD} ; n=5$ ). Bottom panel, data represent quantitative results for top panel results. ${ }^{*}, p<0.05$. 
than those in the previous study [13], as we considered the metronomic approach to target tumor angiogenesis [48-50]. SH003 significantly inhibited VEGF-induced angiogenic processes in vitro including endothelial cell migration, invasion and capillary-like structure formation with no effect on the proliferation. In addition, $\mathrm{SH003}$ at the concentration in this study did not affect pancreatic cancer cell proliferation. Thus, it guarantees that $\mathrm{SH} 003$ is not toxic but effective in the treatment of tumor angiogenesis. Moreover, our in vivo data showed
A

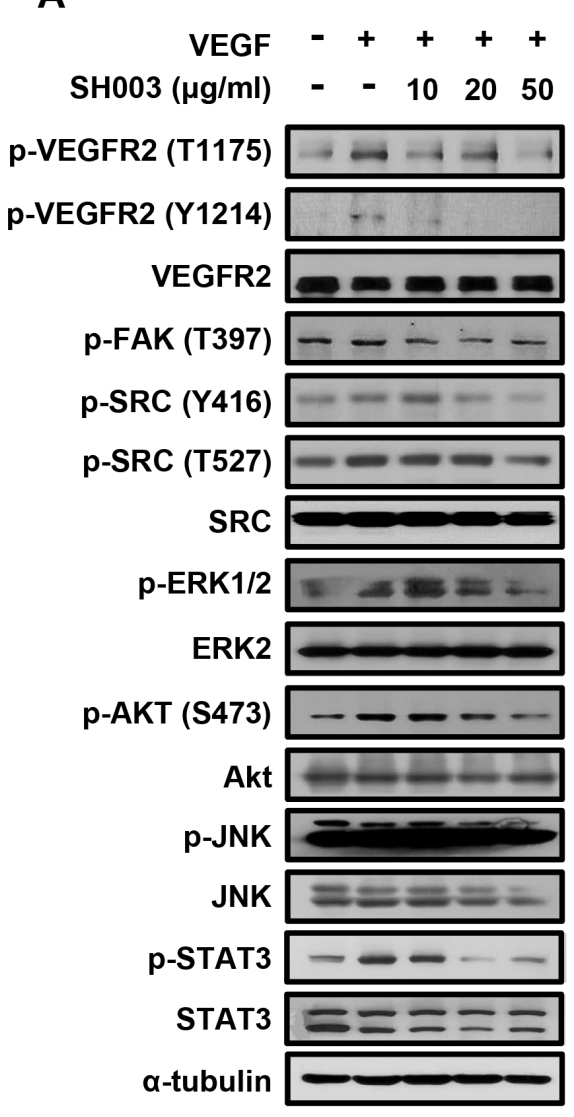

C

$\begin{array}{llllll}\text { VEGF } & - & + & + & + & + \\ \mathrm{SH} 003 & - & - & - & + & +\end{array}$

Time (h) $\quad \begin{array}{lllll}0 & 4 & 24 & 4 & 24\end{array}$

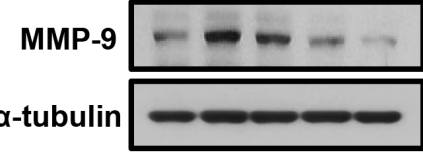

VEGF - + + + +

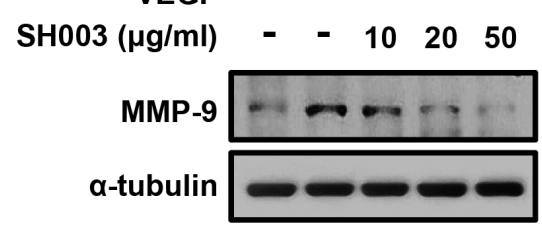

B

VEGF - + + + + + +

$\mathrm{SH} 003--\quad-+++$

Time (min) $0 \quad 306012030 \quad 60120$

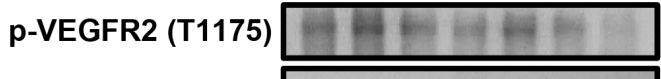

p-VEGFR2 (Y1214)

VEGFR2

p-FAK (T397)

p-SRC (Y416)

p-SRC (T527)

SRC

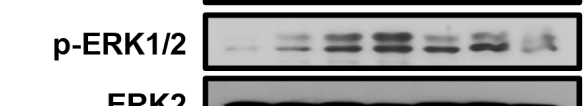

p-AKT (S473)

Akt $-\infty-\infty-\infty$

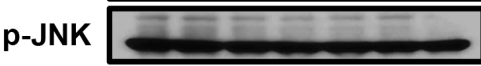

JNK $-2=-2-2$

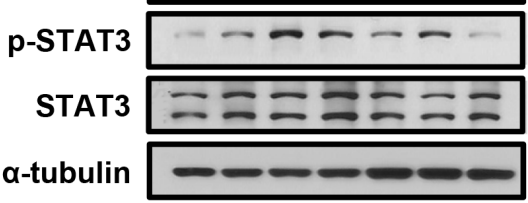

D
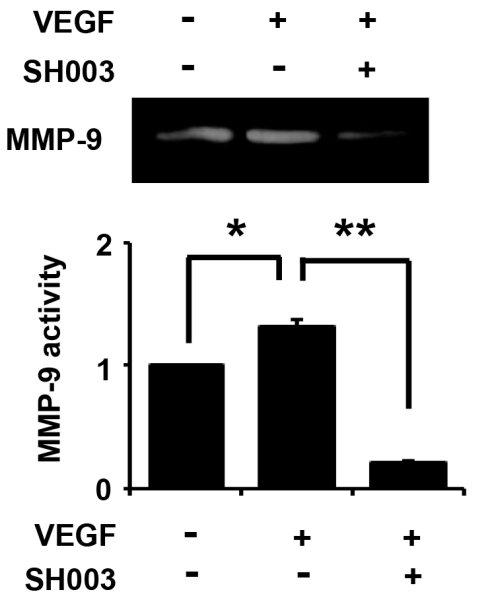

Figure 4: SH003 inhibits VEGF-induced signaling in the endothelial cells. A. SH003 inhibition of VEGF-induced signaling was examined at different concentrations of SH003. B. SH003 inhibition of VEGF-induced signaling was examined for 120 minutes. C. SH003 inhibition of MMP-9 expression was examined at different time points or at concentrations of SH003. D. MMP-9 activity was measured by zymography assay. ${ }^{*}$ and ${ }^{* *}, p<0.05$. 
that $\mathrm{SH} 003$ inhibited xenograft tumor growth as well as VEGF-stimulated vascular permeability. Therefore, our in vitro and in vivo data strongly suggest that $\mathrm{SH} 003$ has antiangiogenic effect.

SH003 blocked VEGF-induced phosphorylation of VEGFR2 by directly interrupting VEGF binding to VEGFR2, resulting in the inhibition of downstream signaling. Moreover, we revealed that components
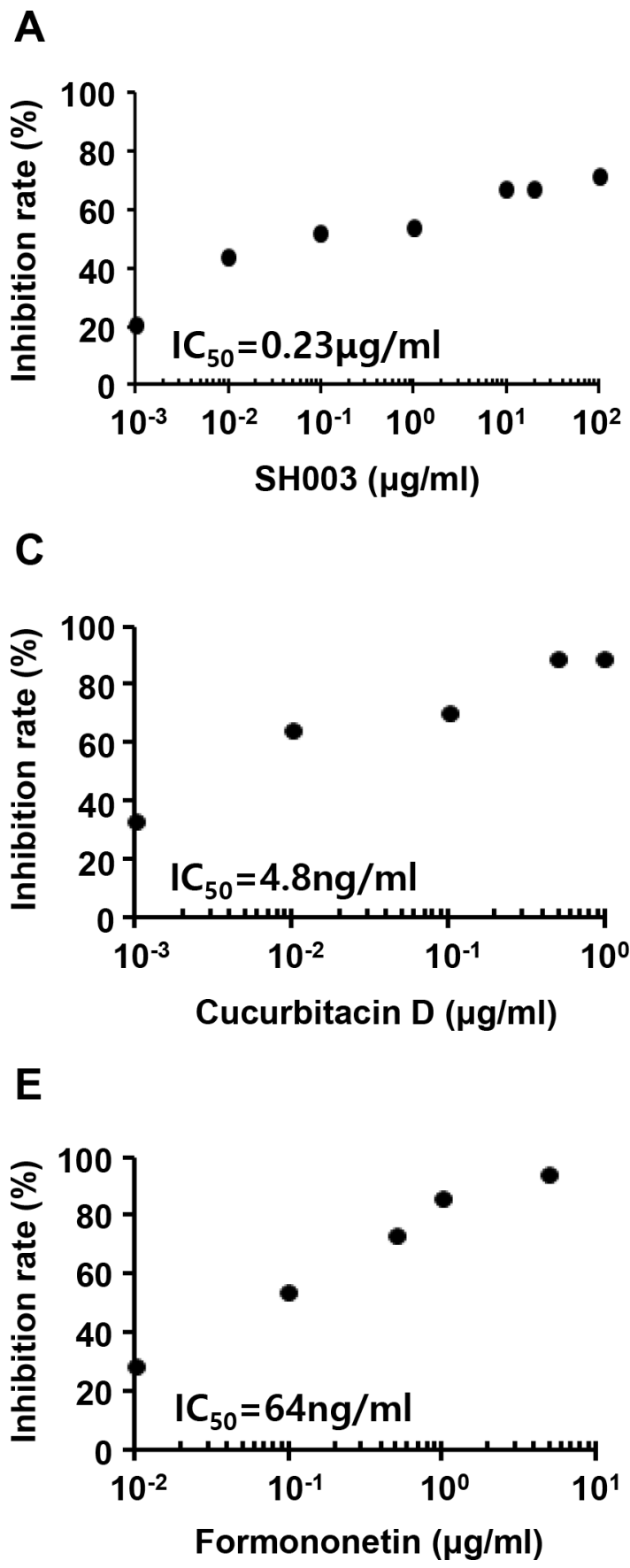

in $\mathrm{SH} 003$ inhibited VEGF/VEGFR2 interaction. While decursin has been revealed to inhibit VEGFR2 phosphorylation [33], this study first shows that chemical components including decursin have abilities to block VEGF binding to VEGFR2. However, it still remains to be elucidated how many chemical components in SH003 interfere with the interaction between VEGF and VEGFR2, and how those components work together as a

B

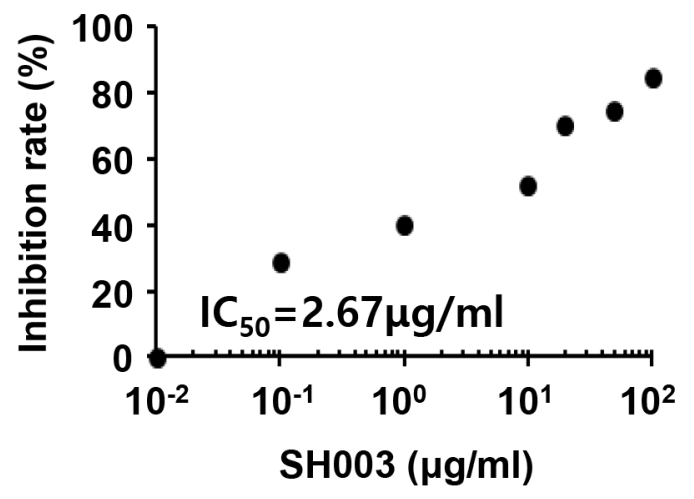

D

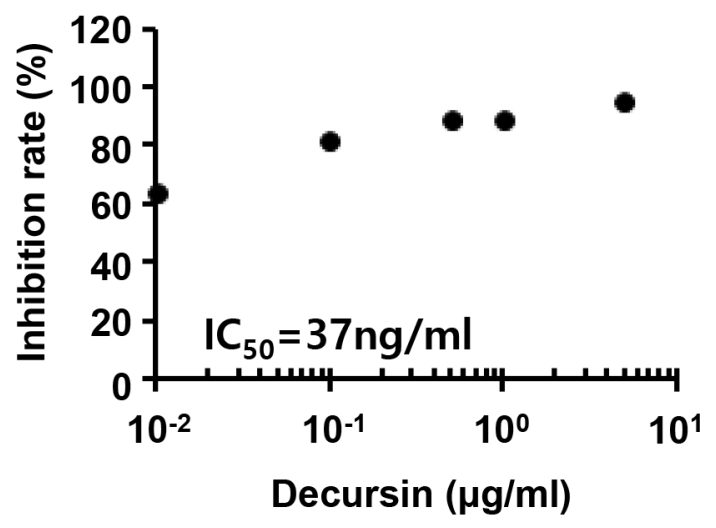

$\mathbf{F}$

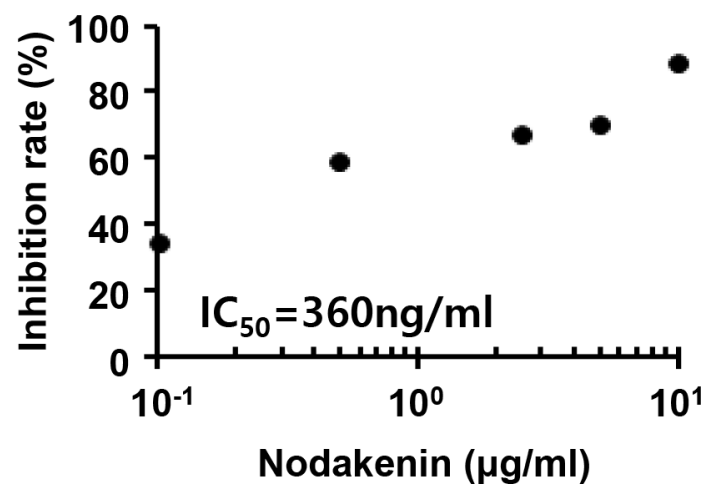

Figure 5: SH003 inhibits VEGF binding to VEGFR2. A-B. SH003 and btVEGF were treated on the plate where recombinant human VEGFR2 or VEGFR1 was coated. C-F. Cucurbitacin D, decursin, formononetin, or nodakenin together with btVEGF were treated on the plate where recombinant human VEGFR2 was coated. 
Table 1: Crude components and amounts of SH003

\begin{tabular}{lccc}
\hline Scientific name & Latin name & Chinese name & Amount (g) \\
\hline Angelica membranaaceus & Astragali Radix & 黃芪 & 333.0 \\
Angelica gigas & Angelicae Gigantis Radix & 當歸 & 333.0 \\
Trichosanthes Kirilowii & Trichosanthis Fructus & 天花粉 & 333.0 \\
Maximowicz & & & \\
\hline
\end{tabular}

whole. Meanwhile, we found that SH003 inhibited VEGF binding to VEGFR1, although SH003 concentrations for the inhibition of VEGF/VEGF1 binding were higher than those for the inhibition of VEGF/VEGFR2 binding. Thus, SH003 effect on VEGF binding to either VEGFR2 or VEGFR1 suggests that SH003 could be applied to other vascular diseases beyond tumor angiogenesis.

This study first revealed that $\mathrm{SH} 003$ as the modified version of TCM inhibits tumor angiogenesis by directly inhibiting VEGF/VEGFR2 interaction. As the concentration of $\mathrm{SHOO3}$ in anti-angiogenesis role was lower than that in anti-breast cancer function, we suggest that metronomic treatment of $\mathrm{SH} 003$ with particular anti-cancer agents could be effective to treat cancer development and metastasis, especially pancreatic cancer. Our ongoing studies include what effective molecules directly inhibits VEGFs/VEGFRs interactions, which will serve better knowledge for TCMs and help understanding vascular diseases.

\section{MATERIALS AND METHODS}

\section{Preparation of SH003 extacts}

SH003 was prepared as described previously [13]. In brief, each component was provided from Hanpoong Pharm and Foods Company (Jeonju, Korea) manufactured by the Good Manufacturing Product (GMP) and mixed as follows: $333 \mathrm{~g}$ of Astragalus membranaceus, $333 \mathrm{~g}$ of Angelica gigas, and 333g of Trichosanthes Kirilowii Maximowicz. The mixture was extracted by $30 \%$ ethanol and then stored at $-80^{\circ} \mathrm{C}$ until use. Table 1 shows information on $\mathrm{SH} 003$ ingredients.

\section{Cell cultures}

Human umbilical vein endothelial cells (HUVECs) were kindly provided by Dr. Kwang Seok Kim (Korea Institute of Radiological and Medical Sciences, Seoul, Korea) and cultured in endothelial cell medium (ECM) with $5 \%$ fetal bovine serum, $1 \%$ endothelial cell growth supplement, and 1\% penicillin/streptomycin solution. Panc-28-luc-luc cells were kindly provided by Dr. Bharat B. Aggarwal (UT-MDA, Houston, USA) and cultured in DMEM with $10 \%$ fetal bovine serum and $1 \%$ penicillin/ streptomycin.

\section{Proliferation, scratching, tube formation, and invasion assay}

For proliferation assays, HUVECs were seeded at $5 \times 10^{3}$ cells in 96-well plates and then exposed to various concentrations of $\mathrm{SH} 003$ in the presence or absence of VEGF (50ng/ml). 5x10 3 Panc-28-luc cells were plated onto 96-well plates and treated with different concentrations of SH003. Cells were incubated for 24 or 72 hours and the cell viability was measured by MTT assay. For scratching assay, HUVECs were plated onto 12well plates, scratched, and then washed with phosphatebuffered saline (PBS). After treatment with SH003 and VEGF $(50 \mathrm{ng} / \mathrm{ml})$ for 9.5 hours, cells migrated toward the wound region were counted. For in vitro tube formation assay, $8 \times 10^{4}$ HUVECs were plated onto matrigel-coated 12 -well plates, and treated with VEGF $(50 \mathrm{ng} / \mathrm{ml})$ and various concentrations of $\mathrm{SH} 003$. After incubation for 9 hours, cells were fixed with $4 \%$ paraformaldehyde and capillary-like structures were measured. For invasion assay, 6x10 ${ }^{4}$ HUVECs were plated onto matrigel-coated $8 \mu \mathrm{m}$ pore size chambers. The upper chambers were filled with different concentrations of $\mathrm{SH} 003$ and the bottom well was added VEGF (50ng/ml) as the chemoattractant. After incubation for 5 7 days, the invaded cells were fixed with $4 \%$ paraformaldehyde and stained with $0.05 \%$ crystal violet. Crystal violet-positive invaded cells were counted. Each experiment was performed in triplicate and repeated three times.

\section{In vivo studies}

All animal experiments were approved by Kyung Hee University Institutional Animal Care and Use Committee (KHU-IACUC). Five-week-old male Balb/c nude mice were purchased from Jungang Lab Animal Inc. (Seoul, Korea). For vascular leakage assays, SH003 $(20 \mu \mathrm{g})$ was injected into the ears or back skins in the presence or absence of VEGF (100ng) for $30 \mathrm{~min}$. 150 $\mu \mathrm{l}$ of $1 \%$ Evans blue dye was injected via tail vein to detect VEGF-induced vascular leakage. The stained tissues were removed and weighed. Evans blue was extracted from the tissues with $100 \mu$ l of formamide solution for 24 hours at $55^{\circ} \mathrm{C}$ and measured spectrophotometrically at $610 \mathrm{~nm}$. For xenograft tumor growth assay, Panc-28-luc cells $\left(1 \times 10^{6}\right)$ mixed with matrigels were subcutaneously 
implanted. For the in vivo bioluminescence imaging analyses, mice were randomly separated into three groups (background, control, and SH003). SH003 (2mg/kg) was orally administrated to $\mathrm{SH} 003$ group, while saline to control group. Mice were injected with $200 \mu \mathrm{l}$ D-luciferin using $25 \mathrm{G}$ syringes and incubated for 60 minutes. The image was captured in NightOWL LB 983 and analyzed using Indigo program (Berthold Technologies, Bad Wildbad, Germany). Body weights and tumor volumes were measured three times a week. Tumor volumes were determined by a formula: volume $=$ length $\mathrm{x}$ width ${ }^{2} \times 0.5$. At the end of experiments, mice were euthanized and then tumors were isolated. For histological analyses, tumors were fixed with $4 \%$ paraformaldehyde and embedded in paraffin. Immunohistochemical staining for Ki-67, CD31, p-VEGFR2 (Y1175), and cleaved caspase-3 were carried out.

\section{Western immunoblotting}

Total $20 \mu \mathrm{g}$ of protein was loaded on $10 \sim 15 \%$ SDS-PAGE, transferred to nitrocellulose membrane and blotted by appropriate antibodies. Anti-p-JNK, -ERK2, and -p-AKT (S473) antibodies were purchased from Santa Cruz Biotechnology (Santa Cruz, CA, USA). AntiAKT, -p-ERK1/2, -JNK, -p-VEGFR2 (Y1175), -MMP9, -VEGFR2, -p-FAK (T397), -p-SRC (Y416), -p-SRC (Y527), -SRC, -p-STAT3, and -STAT3 antibodies were obtained from Cell Signaling Technology (Danvers, MA, USA). Anti-p-VEGFR2 (Y1214) antibody was purchased from R\&D systems (Minneapolis, MN, USA). Anti- $\alpha-$ tubulin antibody was purchased from Sigma (St. Louis, MO, USA).

\section{Zymography assay}

For gelatinolytic activities of samples, cells were treated with VEGF and SH003 for 24 hours and then subjected to zymography. After incubation, medium was collected and concentrated using Amicon Ultra-4 centrifugal filters (Millipore, New York, USA). The concentrated was mixed with non-reducing $5 \mathrm{X}$ sample buffer and electrophoresed in 8\% SDS-PAGE gels containing $0.2 \%$ gelatin. Gels were run at $90 \mathrm{~V}$ for 3 hours at $4{ }^{\circ} \mathrm{C}$ and washed for 40 minutes in $2.5 \%$ Triton $\mathrm{X}-100$ solution at room temperature. The gels were incubated for 20 hours at $37^{\circ} \mathrm{C}$ in $50 \mathrm{mM}$ Tris- $\mathrm{HCl}$ containing 0.15 $\mathrm{M} \mathrm{NaCl}$ and $10 \mathrm{mM} \mathrm{CaCl}_{2}$ (pH 7.8). Next, the gels were stained for 1 hour in $0.05 \%$ Coomassie Brilliant Blue solution and de-stained until clear bands were visible.

\section{In vitro solid-phase binding assay of biotinylated VEGF to recombinant human VEGFR1-2}

The method was performed as described previously [51]. 96-well microplate (Thermo Fisher Scientific,
Waltham, USA) was coated with $100 \mu$ l of phosphate buffer saline (PBS) containing $500 \mathrm{ng} / \mathrm{ml}$ of either VEGFR-1 or $-2 \mathrm{ECD} / \mathrm{Fc}$ chimera (R\&D Systems, Minneapolis, USA). The plate was sealed and incubated overnight at $4{ }^{\circ} \mathrm{C}$. After 3 times washes with $200 \mu$ of PBS containing $0.05 \%(\mathrm{v} / \mathrm{v})$ Tween 20 , the plate was blocked by adding $100 \mu \mathrm{l}$ of PBS with $1 \%(\mathrm{w} / \mathrm{v})$ bovine serum albumin (BSA), and incubated for 2-3 hours at room temperature. The plate was washed 3 times and added with $100 \mu \mathrm{l}$ of diluted standards (biotinylated VEGF (btVEGF), R\&D systems, Minneapolis, USA) or compounds (with 50ng/ml btVEGF) in PBS. After 2.5-3 hours incubation at room temperature, the plate was washed 3 times, and $100 \mu l$ of streptavidin-HRP (R\&D Systems, Minneapolis, USA) was diluted at 1:250 in blocking buffer. The plate was incubated for another 1 hour at room temperature, and then washed five times with $200 \mu$ l of wash buffer with $100 \mu \mathrm{l}$ of substrate solution (BD Biosciences, Sandiego, USA). After 1-3 hours incubation at room temperature, the plate was then added with $50 \mu$ l of stop solution ( $1 \mathrm{M}$ $\mathrm{H}_{3} \mathrm{PO}_{4}$ ) to each well. The signal measured at $450 \mathrm{~nm}$ using ELISA plate reader.

\section{Statistical analysis}

All experimental data were presented as a mean \pm SD and analyzed by Student $t$-test or one-way ANOVA using SPSS software. $P$-value $<0.05$ means statistically significant.

\section{ACKNOWLEDGMENTS}

We thank Kwang Seok Kim at Korea Institute of Radiological and Medical Science (Seoul, Korea) for providing us with HUVECs. Panc-28-luc cells were a kind gift of Dr. Bharat B. Aggarwal at UT-MDA (Houston, TX, USA).

\section{CONFLICTS OF INTEREST}

The authors have declared that the issue is under patent filings.

\section{GRANT SUPPORT}

This work was supported by a grant from Korean Medicine R\&D project of the Ministry Of Health and Welfare (B110043).

\section{REFERENCES}

1. Liu J, Wang S, Zhang Y, Fan HT, Lin HS. Traditional Chinese medicine and cancer: History, present situation, and development. Thorac Cancer. 2015; 6:561-569. 
2. Sun GD, Li CY, Cui WP, Guo QY, Dong CQ, Zou HB, Liu SJ, Dong WP, Miao LN. Review of Herbal Traditional Chinese Medicine for the Treatment of Diabetic Nephropathy. J Diabetes Res. 2016; 2016:5749857.

3. Grayson M. Traditional Asian medicine. Nature. 2011; 480:S81

4. Lee H. Asian medicine: Many unique types. Nature. 2012; 482:35.

5. Xu J, Liu M, Xia Z. Asian medicine: Call for more safety data. Nature. 2012; 482:35.

6. Zha LH, He LS, Lian FM, Zhen Z, Ji HY, Xu LP, Tong XL. Clinical Strategy for Optimal Traditional Chinese Medicine (TCM) Herbal Dose Selection in Disease Therapeutics. Am J Chin Med. 2015:1550086.

7. Xing $\mathrm{Y}, \mathrm{Hu} \mathrm{D}$, Zhang $\mathrm{T}$, Antzelevitch C. Traditional Chinese Medicine and Vascular Disease. Evid Based Complement Alternat Med. 2015; 2015:430818.

8. Huang CY, Lai WY, Sun MF, Lin CC, Chen BC, Lin HJ, Chang CM, Yang CH, Huang KC, Yen HR. Prescription patterns of traditional Chinese medicine for peptic ulcer disease in Taiwan: A nationwide population-based study. $\mathrm{J}$ Ethnopharmacol. 2015; 176:311-320.

9. Wu M, Lu P, Shi L, Li S. Traditional Chinese patent medicines for cancer treatment in China: a nationwide medical insurance data analysis. Oncotarget. 2015; 6:38283-38295. doi: 10.18632/oncotarget.5711.

10. Konkimalla VB, Efferth T. Evidence-based Chinese medicine for cancer therapy. J Ethnopharmacol. 2008; 116:207-210.

11. Jafari S, Abdollahi M, Saeidnia S. Personalized medicine: a confluence of traditional and contemporary medicine. Altern Ther Health Med. 2014; 20:31-40.

12. Hu J, Liu B. The basic theory, diagnostic, and therapeutic system of traditional Chinese medicine and the challenges they bring to statistics. Stat Med. 2012; 31:602-605.

13. Choi YK, Cho SG. Herbal extract SH003 suppresses tumor growth and metastasis of MDA-MB-231 breast cancer cells by inhibiting STAT3-IL-6 signaling. 2014; 2014:492173.

14. Lee DH, Kim SS, Seong S, Kim N, Han JB. Korean medicine therapy as a substitute for chemotherapy for metastatic breast cancer: a case report. Case Rep Oncol. 2015; 8:64-71.

15. Liu C. TA, Yang S. Chinese Herbal Medicine: Modern Applications of Traditional Formulas. (NW Bocca Raton: CRC Press LLC). 2005.

16. Chang HM, Centre CUoHKCMR. Advances in Chinese medicinal materials research: World Scientific. 1985.

17. Yin G, Tang D, Dai J, Liu M, Wu M, Sun YU, Yang Z, Hoffman RM, Li L, Zhang S, Guo X. Combination Efficacy of Astragalus membranaceus and Curcuma wenyujin at Different Stages of Tumor Progression in an Imageable Orthotopic Nude Mouse Model of Metastatic Human Ovarian Cancer Expressing Red Fluorescent Protein. Anticancer Res. 2015; 35:3193-3207.
18. Lee HJ, Lee HJ, Lee EO, Lee JH, Lee KS, Kim KH, Kim $\mathrm{SH}, \mathrm{Lu}$ J. In vivo anti-cancer activity of Korean Angelica gigas and its major pyranocoumarin decursin. Am J Chin Med. 2009; 37:127-142.

19. Kim BS, Seo H, Kim HJ, Bae SM, Son HN, Lee YJ, Ryu S, Park RW, Nam JO. Decursin from Angelica gigas Nakai Inhibits B16F10 Melanoma Growth Through Induction of Apoptosis. J Med Food. 2015; 18:1121-1127.

20. Fang EF, Zhang CZ, Zhang L, Wong JH, Chan YS, Pan WL, Dan XL, Yin CM, Cho CH, Ng TB. Trichosanthin inhibits breast cancer cell proliferation in both cell lines and nude mice by promotion of apoptosis. PLoS One. 2012; 7:e41592.

21. Li M, Li X, Li JC. Possible mechanisms of trichosanthininduced apoptosis of tumor cells. Anat Rec (Hoboken). 2010; 293:986-992.

22. Woo SM, Choi YK, Cho SG, Park S, Ko SG. A New Herbal Formula, KSG-002, Suppresses Breast Cancer Growth and Metastasis by Targeting NF- kappa B-Dependent TNF alpha Production in Macrophages. Evid Based Complement Alternat Med. 2013; 2013:728258.

23. Gensicka M, Glowacka A, Dzierzbicka K, Cholewinski G. Inhibitors of angiogenesis in cancer therapy - synthesis and biological activity. Curr Med Chem. 2015.

24. Huang D, Lan H, Liu F, Wang S, Chen X, Jin K, Mou X. Anti-angiogenesis or pro-angiogenesis for cancer treatment: focus on drug distribution. Int J Clin Exp Med. 2015; 8:8369-8376.

25. Olsson A-K, Dimberg A, Kreuger J, Claesson-Welsh L. VEGF receptor signalling? in control of vascular function. Nat Rev Mol Cell Biol. 2006; 7:359-371.

26. Cines DB, Pollak ES, Buck CA, Loscalzo J, Zimmerman GA, McEver RP, Pober JS, Wick TM, Konkle BA, Schwartz BS, Barnathan ES, McCrae KR, Hug BA, Schmidt A-M, Stern DM. Endothelial Cells in Physiology and in the Pathophysiology of Vascular Disorders. Blood. 1998; 91:3527-3561.

27. Ferrara N, Gerber HP, LeCouter J. The biology of VEGF and its receptors. Nat Med. 2003; 9:669-676.

28. Goel HL, Mercurio AM. VEGF targets the tumour cell. Nat Rev Cancer. 2013; 13:871-882.

29. Chai EZ, Shanmugam MK, Arfuso F, Dharmarajan A, Wang C, Kumar AP, Samy RP, Lim LH, Wang L, Goh BC, Ahn KS, Hui KM, Sethi G. Targeting transcription factor STAT3 for cancer prevention and therapy. Pharmacol Ther. 2016. pii: S0163-7258(15)00185-0. doi: 10.1016/j. pharmthera.2015.10.004.

30. Dong Y, Lu B, Zhang X, Zhang J, Lai L, Li D, Wu Y, Song Y, Luo J, Pang X, Yi Z, Liu M. Cucurbitacin E, a tetracyclic triterpenes compound from Chinese medicine, inhibits tumor angiogenesis through VEGFR2-mediated Jak2-STAT3 signaling pathway. Carcinogenesis. 2010; 31:2097-2104. 
31. Teng LS, Jin KT, He KF, Wang HH, Cao J, Yu DC. Advances in combination of antiangiogenic agents targeting VEGF-binding and conventional chemotherapy and radiation for cancer treatment. J Chin Med Assoc. 2010; 73:281-288.

32. Kamba T, McDonald DM. Mechanisms of adverse effects of anti-VEGF therapy for cancer. Br J Cancer. 2007; 96:1788-1795.

33. Jung MH, Lee SH, Ahn EM, Lee YM. Decursin and decursinol angelate inhibit VEGF-induced angiogenesis via suppression of the VEGFR-2-signaling pathway. Carcinogenesis. 2009; 30:655-661.

34. Oh TW, Park KH, Jung HW, Park YK. Neuroprotective effect of the hairy root extract of Angelica gigas NAKAI on transient focal cerebral ischemia in rats through the regulation of angiogenesis. BMC Complement Altern Med. $2015 ; 15: 101$.

35. Zhang L, Yang Y, Wang Y, Gao X. Astragalus membranaceus extract promotes neovascularisation by VEGF pathway in rat model of ischemic injury. Pharmazie. 2011; 66:144-150.

36. Lei Y, Wang JH, Chen KJ. Comparative study on angiogenesis effect of Astragalus membranaceus and Angelica sinensis in chick embryo choriollantoic membrane [Article in Chinese]. Zhongguo Zhong Yao Za Zhi. 2003; 28:876-878.

37. Son SH, Kim MJ, Chung WY, Son JA, Kim YS, Kim YC, Kang SS, Lee SK, Park KK. Decursin and decursinol inhibit VEGF-induced angiogenesis by blocking the activation of extracellular signal-regulated kinase and c-Jun N-terminal kinase. Cancer Lett. 2009; 280:86-92.

38. Zachary I. VEGF signalling: integration and multi-tasking in endothelial cell biology. Biochem Soc Trans. 2003; 31:1171-1177.

39. Ferrara N. Role of vascular endothelial growth factor in the regulation of angiogenesis. Kidney Int. 1999; 56:794-814.

40. Herkenne S, Paques C, Nivelles O, Lion M, Bajou K, Pollenus T, Fontaine M, Carmeliet P, Martial JA, Nguyen NQ, Struman I. The interaction of uPAR with VEGFR2 promotes VEGF-induced angiogenesis. Sci Signal. 2015; 8:ra117.
41. Vandooren J, Van den Steen PE, Opdenakker G. Biochemistry and molecular biology of gelatinase B or matrix metalloproteinase-9 (MMP-9): the next decade. Crit Rev Biochem Mol Biol. 2013; 48:222-272.

42. Taraboletti G, D'Ascenzo S, Borsotti P, Giavazzi R, Pavan A, Dolo V. Shedding of the matrix metalloproteinases MMP-2, MMP-9, and MT1-MMP as membrane vesicleassociated components by endothelial cells. Am J Pathol. 2002; 160:673-680.

43. Vieira JM, Ruhrberg C, Schwarz Q. VEGF receptor signaling in vertebrate development. Organogenesis. 2010; 6:97-106.

44. Sia D, Alsinet C, Newell P, Villanueva A. VEGF signaling in cancer treatment. Curr Pharm Des. 2014; 20:2834-2842.

45. Clarke JM, Hurwitz HI. Targeted inhibition of VEGF receptor 2: an update on ramucirumab. Expert Opin Biol Ther. 2013; 13:1187-1196.

46. Kwon OS, Jeong MS, Kim B, Kim SH. Antiangiogenic Effect of Ethanol Extract of Vigna angularis via Inhibition of Phosphorylation of VEGFR2, Erk, and Akt. Evid Based Complement Alternat Med. 2015; 2015:371368.

47. Wu Z, Fraley ME, Bilodeau MT, Kaufman ML, Tasber ES, Balitza AE, Hartman GD, Coll KE, Rickert K, Shipman J, Shi B, Sepp-Lorenzino L, Thomas KA. Design and synthesis of 3,7-diarylimidazopyridines as inhibitors of the VEGF-receptor KDR. Bioorg Med Chem Lett. 2004; 14:909-912.

48. Andre N, Carre M, Pasquier E. Metronomics: towards personalized chemotherapy? Nat Rev Clin Oncol. 2014; 11:413-431.

49. Pasquier E, Kavallaris M, Andre N. Metronomic chemotherapy: new rationale for new directions. Nat Rev Clin Oncol. 2010; 7:455-465.

50. Hanahan D, Bergers G, Bergsland E. Less is more, regularly: metronomic dosing of cytotoxic drugs can target tumor angiogenesis in mice. J Clin Invest. 2000; 105:1045-1047.

51. Goncalves V, Gautier B, Garbay C, Vidal M, Inguimbert $\mathrm{N}$. Development of a chemiluminescent screening assay for detection of vascular endothelial growth factor receptor 1 ligands. Anal Biochem. 2007; 366:108-110. 